\title{
Electron Beam Susceptibility of Enteric Viruses and Surrogate Organisms on Fruit, Seed and Spice Matrices
}

\author{
Sophie Butot ${ }^{1} \cdot$ Luca Galbusera $^{1} \cdot$ Thierry Putallaz $^{1} \cdot$ Sophie Zuber ${ }^{1} \mathbb{C}$
}

Received: 15 July 2020 / Accepted: 23 January 2021 / Published online: 10 February 2021

(c) The Author(s) 2021

\begin{abstract}
The objective of this study was to use high-energy electron beam (HEEB) treatments to find surrogate microorganisms for enteric viruses and to use the selected surrogates as proof of concept to investigate low-energy electron beam (LEEB) treatments for enteric virus inactivation at industrial scale on frozen blueberries. Six food matrices inoculated with HAV (hepatitis A virus), MNV S99 (murine norovirus), bacteriophages MS2 and Q $\beta$, and Geobacillus stearothermophilus spores were treated with HEEB at $10 \mathrm{MeV}$ using 4, 8 and $16 \mathrm{kGy}$ doses. G. stearothermophilus spores showed the highest inactivation on all matrices except on raisins, with a dose-dependent effect. HAV reached the maximum measurable $\log _{10}$ reduction $\left(>3.2 \log _{10}\right.$ ) when treated at $16 \mathrm{kGy}$ on raisins. MNV showed the highest resistance of all tested microorganisms, independent of the dose, except on frozen blueberries. On frozen blueberries, freeze-dried raspberries, sesame seeds and black peppercorns, HAV showed a mean inactivation level in between those of MS2 and G. stearothermophilus. Based on this, we selected both surrogate organisms as first approximation to estimate HAV inactivation on frozen blueberries during LEEB treatment at $250 \mathrm{keV}$ using $16 \mathrm{kGy}$. Reductions of 3.1 and $1.3 \log _{10}$ were measured for G. stearothermophilus spores and MS2, respectively, suggesting that a minimum reduction of $1.4 \log _{10}$ can be expected for HAV under the same conditions.
\end{abstract}

Keywords Electron beam $\cdot$ HEEB $\cdot$ LEEB $\cdot$ Enteric viruses $\cdot$ Surrogates $\cdot$ Food

\section{Introduction}

Hepatitis A virus (HAV) and human norovirus (hNoV) are responsible for food-borne outbreaks linked to fresh produce, ready-to-eat foods and shellfish worldwide (Alegbeleye et al. 2018; Bosch et al. 2018; Miranda and Schaffner 2019). Outbreaks are generally associated with food that undergoes minimal processing, such as oysters, fresh-cut lettuce, frozen berries and very recently dried dates (Ethelberg et al. 2010; Tavoschi et al. 2015; Thebault et al. 2013; Anonymous 2018). Consumer demands for minimally processed foods with fresh-like quality are on the rise and food processes must adapt to the preferences, acceptance and needs of the consumer (Knorr and Watzke 2019). In this context, innovative approaches are needed to minimize the risk of viral food-borne outbreaks while retaining good food quality. Non-thermal processes, e.g. high hydrostatic

Sophie Zuber

sophie.zuber@rdls.nestle.com

1 Nestlé Research, Institute of Food Safety and Analytical Science, 1000, 26 Lausanne, Switzerland pressure (HHP), ultra violet light (UV-C), cold plasma and irradiation, have the potential to achieve this goal (Knorr and Watzke 2019). However, virus inactivation by UV-C light is very efficient in clear liquids such as water (Tree et al. 2005; Wiedenmann et al. 1993), but of limited use on complex food surfaces such as berries (Butot et al. 2018). Virus inactivation by HHP is only applicable to high moisture foods, such as oysters and fruit purees (DiCaprio et al. 2019; Kingsley and Chen 2009). For fragile or dry foods, such as berries, herbs and spices, other technologies need to be explored.

Irradiation appears to be a good candidate technology, but data describing the effectiveness of irradiation on food against viruses, especially HAV, are scarce. Besides X-ray, two major irradiation technologies have been explored, gamma irradiation and high-energy electron beam (HEEB) (Farkas 1998; Moosekian et al. 2012; Pillai and Shayanfar 2017; Ravindran and Jaiswal 2019). Food matrix is likely to provide increased survival for viruses during irradiation, but a major hurdle when performing inactivation studies with viruses on food is the low recovery efficiency which is often encountered, leading to a low maximum measurable 
$\log _{10}$ reduction (Bosch et al. 2018; Butot et al. 2014). For example, at a HEEB dose of 12 kilo Gray (kGy), inactivation of MNV (murine norovirus used as hNoV surrogate) in phosphate-buffered saline (PBS) reached $6.4 \log _{10}$, while on strawberries a maximum reduction of $2.2 \log _{10}$ was observed (Sanglay et al. 2011). Similarly, after a $11.9 \mathrm{kGy}$ treatment in simple medium (DMEM) a $4.25 \log _{10}$ reduction of Tulane virus (TV) was measured, compared to a $2.1 \log _{10}$ reduction after a treatment of $12.2 \mathrm{kGy}$ on whole strawberries (DiCaprio et al. 2016). The $D_{10}$ values (dose required to reduce virus titer by $1 \log _{10}$ ) of rotavirus and poliovirus on lettuce were 1.0 and $2.3 \mathrm{kGy}$, respectively, suggesting virusdependent susceptibilities to HEEB (Espinosa et al. 2012). Using gamma irradiation, $D_{10}$ values determined for HAV in lettuce and strawberries were 2.7 and $3.0 \mathrm{kGy}$, respectively (Bidawid et al. 2000), while the HEEB dose required to reduce HAV by $1 \log _{10}$ in whole oysters was $4.8 \mathrm{kGy}$ (Praveen et al. 2013).

A new approach to irradiation processing of food, called low-energy electron beam (LEEB), uses electrons with energies below 300 kilo electron Volt $(\mathrm{keV})$, associated with a very limited penetration ability of around $500 \mu \mathrm{m}$ (Gryczka et al. 2018; Hayashi et al. 1998; Pillai and Shayanfar 2017). In contrast, HEEB uses electrons with energies between 5 and 10 Mega electron Volt $(\mathrm{MeV})$ which penetrate foods with high water content up to $3.9 \mathrm{~cm}$ (Farkas 2006) and has to be carried out at a dedicated irradiation facility with appropriate safety measures. In contrast, the LEEB technology is scalable to continuous processes and can be easily implemented in existing processing lines and offers benefits in cost reduction, environmental performance, and production flexibility (Zhang et al. 2018; Tetra Pak 2019). As such, LEEB is an emerging irradiation technology that performs surface and subsurface decontamination with a minimal influence on food quality (Hertwig et al. 2018). Since microorganisms reside mostly on the surface and subsurface of food if internalized through pores or damaged tissue, the irradiation of the external layer should be sufficient to eliminate food-borne microorganisms (De Lara et al. 2002; Hayashi et al. 1998). For example, Kikuchi et al. recommended LEEB treatment over gamma irradiation for soybean decontamination, because it induces minimum or no quality deterioration, since the electrons do not reach the internal matrix (Kikuchi et al. 2003). Similarly, LEEB was proposed as a method for microbiological decontamination of seeds, as it was shown not to negatively influence germination (Fan et al. 2017). The response of Bacillus pumilus spores was found to be very similar when treated with HEEB and LEEB (Tallentire et al. 2010). Another study investigating Geobacillus and Bacillus spores also showed that the spore inactivation efficiency by LEEB was comparable to that of other ionizing radiations (Zhang et al. 2018). LEEB was also shown to reduce mould spores even on challenging food surfaces such as raisins (Etter et al. 2018). Inactivation of enveloped viruses such as influenza $\mathrm{A}$ in liquids has been demonstrated (Etter et al. 2018), but no data are available on the effect LEEB has on non-enveloped enteric viruses such as HAV and hNoV. The only industrial scale system currently available to the food industry is the Bühler AG Laatu system. As this system is an open system, it does not allow safe handling of pathogens and non-pathogenic surrogates are needed to estimate virus inactivation by LEEB.

The objective of this study was first to use HEEB treatments to find surrogate microorganisms for enteric viruses (HAV and hNoV) which are easy to grow to high titers and use at industrial scale such as bacterial spores or bacteriophages and second to use the surrogate organisms as proof of concept to investigate LEEB treatments for enteric virus inactivation at industrial scale using frozen blueberries as a model matrix.

\section{Materials and methods}

\section{HEEB Irradiation Treatment and Dose Evaluation}

HEEB treatments $(10 \mathrm{MeV})$ were performed at the LEONI Studer AG irradiation centre (Daeniken, Switzerland). Inoculated food samples were treated at three radiation doses, 4 , 8 and $16 \mathrm{kGy}$, to define critical dosages and screen for adequate surrogates. Pretests were performed for the different food matrices used in this study to verify dose distribution within the load. Based on the results, samples were packaged in $50 \mathrm{~mL}$ Falcon tubes with a maximum diameter of $3 \mathrm{~cm}$ which ensured equal irradiation doses throughout the samples. The absorbed doses were measured using alanine pellets (Aerial CRT, France) which were treated together with the samples (nine independent treatments performed on three separate days) and the doses were calculated by comparison with a standard calibration curve established by Aerial CRT onsite at LEONI Studer AG irradiation centre and traceable to NPL standard. The following mean values and standard deviations were obtained: $3.91 \pm 0.03,7.89 \pm 0.14$ and $15.85 \pm 0.33 \mathrm{kGy}$. The results showed that the values for HEEB are close to the nominal doses, and thus, the nominal values are used in the graphs.

\section{LEEB Irradiation Treatment and Dose Evaluation}

LEEB treatments were performed with Laatu designed by Bühler AG (Uzwil, Switzerland). Inoculated frozen blueberries were treated twice with $250 \mathrm{keV}$ to reach a nominal dose of $16 \mathrm{kGy}$. Samples were conveyed via an inlet channel and feeder table which control the throughput and distribution of the product. The berries were treated during free-fall through the treatment zone which consists of two LEEB lamps facing 
each other (https://digital.buhlergroup.com/laatu/). The dose was measured with Riso B3 radiochromic film sensor provided by Riso High Dose Reference Laboratory (HDLR) (Roskilde, Denmark). Due to the practical limitations of placing a dosimeter on the frozen blueberries, a B3 film strip of $230 \mathrm{~mm} \times 20 \mathrm{~mm}$ was attached along a polymer plate covering the entire width of the treatment zone. The dosimeters were exposed to the same beam conditions (voltage, current) as the inoculated frozen blueberries. The dosimetry system (including radiochromic film, sensor measurement software and calibration) is traceable to international standards in line with ISO/ASTM 51261 (ISO 2013). Dose measurements using Riso B3 films determined a mean surface dose value of $15.51 \pm 1.31 \mathrm{kGy}$ which is close to the nominal dose, and thus, the nominal value is used in the graph.

\section{Food Matrices and Pretreatments}

Six different food matrices were used in this study. Pumpkin, and sesame seeds were kindly provided by the German Institute of Food Technologies (DIL) (Quakenbrück, Germany), black peppercorns, fresh blueberries, freeze-dried raspberry flakes and raisins were purchased at Sabater (Spain), a local distributor (Lausanne, Switzerland), Chaucer (UK) and Mariani Packing (USA), respectively. X-ray pretreatments (25-50 kGy) of the matrices to kill background microflora before inoculation with target organisms were performed at Synergy Health Daeniken AG (Daeniken, Switzerland).

\section{Geobacillus Spore Suspension}

Ready to use suspension of Geobacillus stearothermophilus (ATCC 7953) spores, containing 7.7-8.7 $\log _{10}$ colony forming units $(\mathrm{CFU}) / \mathrm{mL}$ of viable spores, was purchased from Merck Millipore (Burlington, USA) and was used to inoculate the different food matrices.

\section{Viruses and Preparation of Suspensions}

The cytopathogenic HAV strain HM-175 (ATCC VR-1402), the murine norovirus strain MNV S99 used as proxy for $\mathrm{hNoV}$ and provided courtesy of the Friedrich-Loeffler-Institut in Germany (Mueller 2007) were propagated, assayed and titrated on FRhK-4 cells (ATTC CRL1688) and RAW 264.7 cells (ATCC TIB-71), respectively, as described previously (Butot et al. 2018). Viral stock titers were 6.6 and $7.6 \log _{10}$ $50 \%$ tissue culture infective dose $\left(\mathrm{TCID}_{50}\right) / \mathrm{mL}$ for $\mathrm{HAV}$ and MNV, respectively. MS2 bacteriophage (ATCC 15597-B1) was propagated, assayed and enumerated in Escherichia coli K12 (ATCC 23631), as described previously (Butot et al. 2018). The Q $\beta$ bacteriophage (ATTC 23631-B1) was propagated, assayed and enumerated using the same protocols as for MS2. The MS2 and Q $\beta$ stock concentrations were 11.3 and $10.1 \log _{10}$ plaque forming units $(\mathrm{PFU}) / \mathrm{mL}$, respectively.

\section{Inoculation of Food Matrices with Bacteria and Viruses}

Suspension $(100 \mu \mathrm{L})$ of bacterial spores, viruses or bacteriophages were spotted (droplets of $5.2 \pm 0.4 \mu \mathrm{L}$ ) directly on the food surface of the seven selected food matrices (pumpkin, and sesame seeds, peppercorns, fresh blueberries, freezedried raspberries and raisins) using a technique described previously (Delbeke et al. 2015). For the HEEB treatment, $10 \mathrm{~g}$ of each food matrix were inoculated with bacterial spores, viruses or bacteriophages, respectively, except for the freeze-dried raspberries, for which $5 \mathrm{~g}$ was used. The contaminated samples were allowed to dry in a biosafety cabinet at room temperature for $1 \mathrm{~h}$, before being packaged in a $50 \mathrm{~mL}$ tube and subjected to the HEEB treatment. The inoculated fresh blueberry samples were frozen at $-20{ }^{\circ} \mathrm{C}$ after the packaging. Following the same approach, blueberry samples were inoculated with G. stearothermophilus and MS2 bacteriophages and frozen prior to the LEEB treatment.

\section{Recovery of Contaminants from Food Matrices}

To recover inoculated spores of G. stearothermophilus from matrices, 5 or $10 \mathrm{~g}$ sample was aseptically transferred to a stomacher filter bag, diluted 1:10 in buffered peptone water and stomached for $1 \mathrm{~min}$. From this initial suspension, $10 \mathrm{~mL}$ was submitted to a heat treatment at $80{ }^{\circ} \mathrm{C}$ for $10 \mathrm{~min}$ to allow the spores to germinate. After the heat treatment, tubes were cooled down in cold water. Serial tenfold dilutions in tryptone salt solution were performed and plated on Plate Count Agar supplemented with $1 \mathrm{~g} / \mathrm{L}$ of soluble starch and incubated at $55{ }^{\circ} \mathrm{C} \pm 1{ }^{\circ} \mathrm{C}$ for $48 \mathrm{~h} \pm 4 \mathrm{~h}$. The limit of quantification (LOQ) was $2.00 \log _{10} \mathrm{CFU} / 10 \mathrm{~g}$ for all food matrices except for the freeze-dried raspberries, for which the LOQ was $1.98 \log _{10} \mathrm{CFU} / 5 \mathrm{~g}$.

HAV and MNV were recovered from 5 or $10 \mathrm{~g}$ samples using the ISO 15,216 virus extraction method (soft fruits protocol) with slight modifications: no added process control, as untreated samples were analyzed to determine recovery rate and the chloroform/butanol clarification step was not performed (ISO 2017). Before enumeration, concentrated samples were decontaminated by sequential filtering through $0.45 \mu \mathrm{m}$ and then $0.22 \mu \mathrm{m}$ spin centrifuge tube filters (Corning, New York) pretreated with $300 \mu \mathrm{L}$ of phosphate-buffered saline ( $\mathrm{pH} 7.2 \pm 0.2$ ) containing $10 \%$ fetal calf serum followed by $\mathrm{TCID}_{50}$ titration as previously described (Butot et al. 2018). The LOQ was $1.05 \log _{10} \mathrm{TCID}_{50} / 10 \mathrm{~g}$ for all food matrices except for the freeze-dried raspberries, for which the LOQ was $1.35 \log _{10} \mathrm{TCID}_{50} / 5 \mathrm{~g}$ as the pellet obtained after the polyethylene glycol precipitation was 
too viscous and therefore resuspended in $1 \mathrm{~mL}$ instead of $0.5 \mathrm{~mL}$.

MS2 and Q $\beta$ were recovered from 5 or $10 \mathrm{~g}$ samples using the protocol described previously (Butot et al. 2018). Briefly, the sample was transferred in a filter bag containing $50 \mathrm{~mL}$ of buffer $(100 \mathrm{mM}$ Tris, $50 \mathrm{mM}$ glycine, $3 \%(\mathrm{~m} / \mathrm{v})$ beef extract and $50 \mathrm{mM} \mathrm{MgCl}_{2}$ and adjusted to $\mathrm{pH} 9.5$ with $\mathrm{NaOH}$ solution) and stomached for $1 \mathrm{~min}$. Serial dilutions (tenfold) were prepared and virus titer was quantified using ISO method 10705-1:1995(E), Annex C (ISO 1995). The LOQ was $2.78 \log _{10}$ PFU/10 $g$ for all food matrices except for the freeze-dried raspberries, for which the LOQ was 2.74 $\log _{10}$ PFU/5 g.

For all matrix/strain combinations, untreated inoculated triplicate samples were analyzed to determine the $\log _{10}$ reductions, the maximum measurable $\log _{10}$ reduction calculated as $\log _{10}\left(\mathrm{LOQ} / N_{0}\right)$ and the recovery rates and uncontaminated triplicate samples were analyzed as negative controls following the procedures described above.

\section{Statistical Analysis}

Reductions in spore and infectious viral particle counts (inactivation) were calculated as $\log _{10}\left(N_{x} / N_{0}\right)$, where $N_{x}$ is the spore count or the viral titer recovered from treated food matrices and $N_{0}$ is the initial count or titer recovered from untreated food matrices (mean of three replicates). Values of $N_{x}$ below the LOQ were entered as being at that limit and this leads the $\log _{10}$ reduction to reach the maximum measurable $\log _{10}$ reduction.

A $t$ test with a significance level at 0.05 and without correction for multiplicity of test was used to test whether the mean $\log _{10}$ reduction of the replicates in one condition was statistically different from the mean in another condition. Furthermore, it is also important to understand if the single measurements are consistently higher or lower. Thus, for each condition we also computed a prediction interval, corresponding to the interval where $95 \%$ of $\log _{10}$ reductions measured in future experiments are expected to fall. If the prediction intervals of two conditions are non-overlapping, we can be confident that not only the mean $\log _{10}$ reductions are different, but also that the single observations in future experiments are going to be well separated.

In order to compute the $t$ tests and the prediction intervals, the variance inside each condition is needed. However, as the maximum measurable $\log _{10}$ reduction was reached in some conditions, the measured values are not representative of the real ones and must be discarded in the computation of the variance. This leads to having conditions with very few points (possibly zero) from which to estimate the variance. To solve this issue, we assumed that the variance in the $\log _{10}$ reductions of a microorganism in a given matrix is independent from the dose. This allows us to use ANOVA on the values above maximum measurable $\log _{10}$ reduction to compute a pooled variance, common to all the measurements of a given microorganism in a given matrix. Notice that since the width of the prediction interval depends only on the variance of the observations, the prediction intervals have the same width for all the doses of a given microorganism and matrix. Finally, the mean log reductions computed in the presence of values at the maximum measurable $\log _{10}$ reduction are just a lower bound, since the real log reductions might be higher. This means that the presence of values at the maximum measurable $\log _{10}$ reduction can hide the effectiveness of the treatment, since the real mean $\log$ reduction might actually be bigger than the estimated one. However, this does not affect the size of the prediction intervals, which are computed using only values above the maximum measurable $\log _{10}$ reduction.

We notice that the overall variability of the data is determined by different sources, for example, a variability in the recovery rates and in the irradiation values, and a biological variability in the response of the microorganisms. Therefore, the variance computed as described before takes into account all these sources of variability, that are also likely to be found when applying the irradiation technology to real production samples.

All statistical analyses were performed with R v3.6.1 (R-Core Team 2019).

\section{Results}

\section{Recovery Efficiencies}

To evaluate the efficiency of the methodologies employed, the recovery of each microorganism was determined for all matrices tested as the ratio, in percentage, of $N_{0}$ which corresponds to the initial count recovered from untreated matrices and the calculated theoretical count using the stock concentration and the volume inoculated (Table 1). G. stearothermophilus was recovered efficiently with mean values ranging between 46.61 and $100.79 \%$ for all matrices,

Table 1 Recovery efficiencies of each microorganism-matrix combination expressed in percentage $(\%)$

\begin{tabular}{lccccc}
\hline & Geobacillus & HAV & MNV & MS2 & Q $\beta$ \\
\hline Frozen blueberries & 100.79 & 0.65 & 0.01 & 1.59 & 0.04 \\
Freeze-dried raspberries & 25.72 & 0.40 & - & 0.03 & 0.05 \\
Raisins & 92.86 & 4.22 & 0.06 & 0.09 & 2.97 \\
Peppercorns & 82.54 & 0.89 & 16.24 & 0.80 & 0.03 \\
Sesame seeds & 46.61 & 0.46 & 1.35 & 9.92 & 17.76 \\
Pumpkin seeds & 63.92 & 0.12 & 0.60 & 0.06 & 7.26 \\
\hline
\end{tabular}

- the missing value could not be determined due to technical issues 
except for the freeze-dried raspberries where a rate of $25.72 \%$ was obtained. On the opposite, the bacteriophages and the viruses showed lower recovery rates. The bacteriophages were recovered with efficiencies ranging from 0.03 to $9.92 \%$ for MS2 and $0.03-17.76 \%$ for $\mathrm{Q} \beta$. Matrix effects were observed but no correlation was found between the two bacteriophages. Recovery rates above $1 \%$ were obtained with sesame seed and frozen blueberry matrices for MS2 and with sesame seed, pumpkin seed and raisin samples for $\mathrm{Q} \beta$. For viruses, the recovery rates oscillated from 0.12 and $4.22 \%$ for HAV and from 0.01 to $16.24 \%$ for the $\mathrm{hNoV}$ proxy MNV. Recoveries above $1 \%$ for HAV were reached only on raisins and for MNV only on sesame seeds and peppercorns. The freeze-dried raspberries showed low recoveries for all the microorganisms (Table 1). In addition, this food product induced a cytotoxic effect on the RAW 264.7 cells, making it impossible to report inactivation results for MNV. However, this effect was not observed on the FrhK4 cells used to measure the infectivity of HAV.

The recovery efficiency is an important parameter as it has an impact on the maximum measurable $\log _{10}$ reduction. Indeed, the lower the recovery, the lower the $N_{0}$ and the maximum measurable $\log _{10}$ reduction will be. As an example, $5.45 \log _{10} \mathrm{TCID}_{50}$ of HAV were inoculated on $5 \mathrm{~g}$ freeze-dried raspberries, but with a recovery efficiency of $0.40 \%$, only $3.04 \log _{10} \mathrm{TCID}_{50} / 5 \mathrm{~g}$ of HAV were enumerated on the samples not treated $\left(N_{0}\right)$. Therefore, taking into consideration this $N_{0}$ value and the LOQ of $1.35 \log _{10}$
$\mathrm{TCID}_{50} / 5 \mathrm{~g}$, the maximum measurable $\log _{10}$ reduction was $1.69 \log _{10}$.

We notice that a low recovery rate limits the maximum measurable $\log _{10}$ reduction, therefore decreasing the power of detecting strong reductions. However, even for the treatments where the low recovery leads to values below the LOQ, meaningful conclusions can still be drawn, as will be discussed in the following sections. Also, the low variability rates can increase the variance of the measured effects. However, as explained in "Materials and Methods" section, the computation of the $t$ tests and of the prediction intervals take into account also any variation in the recoveries.

\section{Reductions of HAV and MNV By HEEB Treatment on Different Food Matrices}

Inactivation values of HAV and MNV inoculated onto the different model food matrices (fruit, spice and seed) are shown in Fig. 1. The results are shown in $\log _{10}$ reduction with $95 \%$ prediction intervals (not confidence intervals) to better represent the "minimal" effect of a certain treatment condition. A measure obtained from a future experiment will fall on this interval with a probability of $95 \%$. The highest reduction of HAV was observed when inoculated on raisins (Fig. 1). On this matrix at $8 \mathrm{kGy}$, a mean reduction of $1.9 \log _{10}$ was measured, ensuring a minimal reduction of only $0.7 \log _{10}$, according to the $95 \%$ prediction interval. At $16 \mathrm{kGy}$ the maximum measurable $\log _{10}$ reduction was reached $\left(>3.2 \log _{10}\right)$, corresponding to a minimal reduction

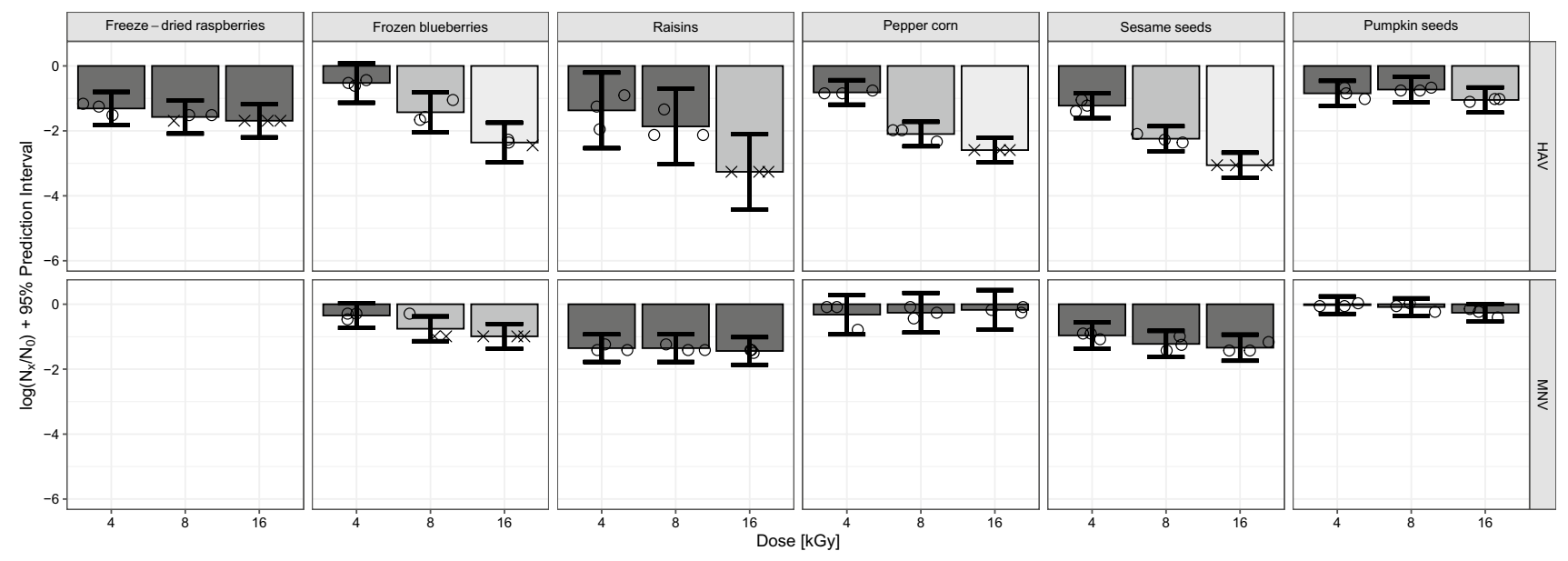

Fig. 1 Mean inactivation of HAV and MNV on freeze-dried raspberries, frozen blueberries, raisins, pepper corns, sesame seeds and pumpkin seeds treated with HEEB at 4,8 and $16 \mathrm{kGy}$. Different shadings show significant differences in the mean $\log _{10}$ reductions between dosages within each microorganism-matrix combination. Bars represent $95 \%$ prediction intervals. Open circles represent single values, crosses represent single values below the maximum measurable $\log _{10}$ reduction. To compute the mean $\log$ reduction, both circles and crosses have been taken into account. For this reason, in the presence of values below the maximum measurable $\log _{10}$ reduction, we only have a lower bound on the mean reduction. For the prediction intervals, only the circles have been considered, and a common variance has been computed for a given microorganism-matrix combination. Therefore, all the prediction intervals for a given combination have the same widths, and they are not affected by having values below the maximum measurable $\log _{10}$ reduction. Supplementary Table 1 contains the raw data used to generate Fig. 1 
which is at least $2 \log _{10}$ as given by the prediction interval. Notice that we talk about minimal reduction, because the real mean reduction might be bigger than the estimated one, as the maximum measurable $\log _{10}$ reduction has been reached for all the three replicates (this does not affect the size of the prediction intervals, which are computed only from values above the maximum measurable $\log _{10}$ reduction). A dose-dependent inactivation of HAV was also observed on frozen blueberries with mean reduction values of $0.5,1.4$ and $2.4 \log _{10}$ at 4,8 and $16 \mathrm{kGy}$, respectively. For comparison with raisins, the minimal $\log _{10}$ reduction at $16 \mathrm{kGy}$ on frozen blueberries was estimated at $1.75 \log _{10}$. The reductions of HAV inoculated on freeze-dried raspberries subjected to the three HEEB dosages of 4, 8 and $16 \mathrm{kGy}$ appeared not significantly different from each other (bars of the same colour for all three dosages), but this may be an artefact which could be overcome if we had a better extraction efficiency allowing to see a greater maximum measurable $\log _{10}$ reduction than $1.7 \log _{10}$ at $16 \mathrm{kGy}$. A significant dose-dependent inactivation of HAV subjected on HEEB treatment of 4, 8 and $16 \mathrm{kGy}$ was seen on peppercorns and sesame seeds, with mean HAV reductions of 2.1 and 2.2 $\log _{10}$ when treated at $8 \mathrm{kGy}$, respectively. At $16 \mathrm{kGy}$ the maximum measurable $\log _{10}$ reduction was reached on both matrices, corresponding to $2.6 \log _{10}$ for peppercorns and 3.1 $\log _{10}$ for sesame seeds. The reductions of HAV inoculated on pumpkin seeds were lower than the reductions observed on sesame seeds (a mean inactivation of only $1.1 \log _{10}$ at $16 \mathrm{kGy}$ ) which may indicate complex matrix effects and show that predictions of inactivation are risky, even for similar food matrices.

Compared to HAV, MNV showed a higher resistance to HEEB on all matrices, except for frozen blueberries where maximum measurable $\log _{10}$ reductions were reached in most cases, preventing a meaningful comparison (Fig. 1). This highlights the high resistance of MNV to irradiation by HEEB on food matrices (complementary Table 1). The inactivation was lowest on pumpkin seeds and peppercorns with mean inactivation values showing no significant differences among the three dosages applied and ranging from 0.03 to $0.32 \log _{10}$. MNV inoculated on raisins and sesame seeds did not show significant dose-dependent differences either, with reductions ranging from 1.35 to $1.44 \log _{10}$ on raisins and 0.96 and $1.34 \log _{10}$ on sesame seeds. A significantly higher inactivation at a higher HEEB dose could only be measured for MNV on frozen blueberries with mean log reductions of $0.35,>0.76$ and $>1.18 \log _{10}$ for treatments at 4,8 and $16 \mathrm{kGy}$, respectively.

\section{Reductions of Selected Surrogate Organisms By HEEB Treatment}

Inactivation values of the three surrogate organisms (spores G. stearothermophilus and bacteriophages MS2 and Q $\beta$ ) inoculated onto the different model food matrices (fruit, spice and seed) are shown in Fig. 2. MS2 and Q $\beta$ both showed a high resistance to HEEB treatments compared to G. stearothermophilus, with mean reductions generally lower than $1.5 \log _{10}$. The only exceptions were raisins, where both bacteriophages showed reductions close to those for $G$. stearothermophilus. We noticed that for some matrices, the prediction intervals at two different irradiation doses (error bars) were overlapping, meaning that the doses do not have a statistically different effect. That is, we cannot conclude that future measurements at the higher dose will have a higher log reduction compared to the lower dose. The overlapping of prediction intervals can happen even when the means are statistically different (the bars have a different shade of grey), as for example for $\mathrm{Q} \beta$ in sesame seeds treated at 4 and $8 \mathrm{kGy}$. This shows that, although the mean reductions can be different at two different irradiation doses, for single future measurements we cannot conclude that the reduction is lower at $4 \mathrm{kGy}$ compared to $8 \mathrm{kGy}$.

On sesame seeds, however, MS2 showed a clear lower reduction compared to $\mathrm{Q} \beta$, both considering the mean and the prediction interval (Supplementary Table 2). Given the similarities between the two bacteriophages, and the fact that compared to $\mathrm{Q} \beta$, MS2 showed a higher recovery and a dose-dependent reduction on frozen blueberries, we chose to focus only on MS2 as candidate surrogate for HAV and hNoV for the LEEB study. This choice is especially relevant for HAV, as on all food matrices and doses, HAV showed a mean reduction which was either higher or non-statistically different than the mean reduction for MS2 (Supplementary Table 3).

Geobacillus stearothermophilus showed a dose-dependent inactivation on all tested matrices with a generally higher inactivation than the tested viruses, for both mean reductions and prediction intervals, except on raisins (Fig. 2). On frozen blueberries, for example, a 1.3, 3.3 and $>5.6 \log _{10}$ reduction was measured for HEEB treatments at 4,8 and $16 \mathrm{kGy}$, respectively. In comparison, at $16 \mathrm{kGy}$ on the same matrix, $2.4 \log _{10}$ and $1.8 \log _{10}$ reductions were measured for HAV and MS2, respectively (Figs. 1, 2). Similarly, on sesame seeds, pumpkin seeds and peppercorns at 4,8 and $16 \mathrm{kGy}, \mathrm{HAV}$ showed a lower mean inactivation level compared to G. stearothermophilus (Figs. 1, 2). This intermediate ranking of HAV between G. stearothermophilus spores and MS2 determined by HEEB was ideal to select both surrogate organisms as first approximation to estimate HAV inactivation during LEEB treatments of frozen blueberries. 


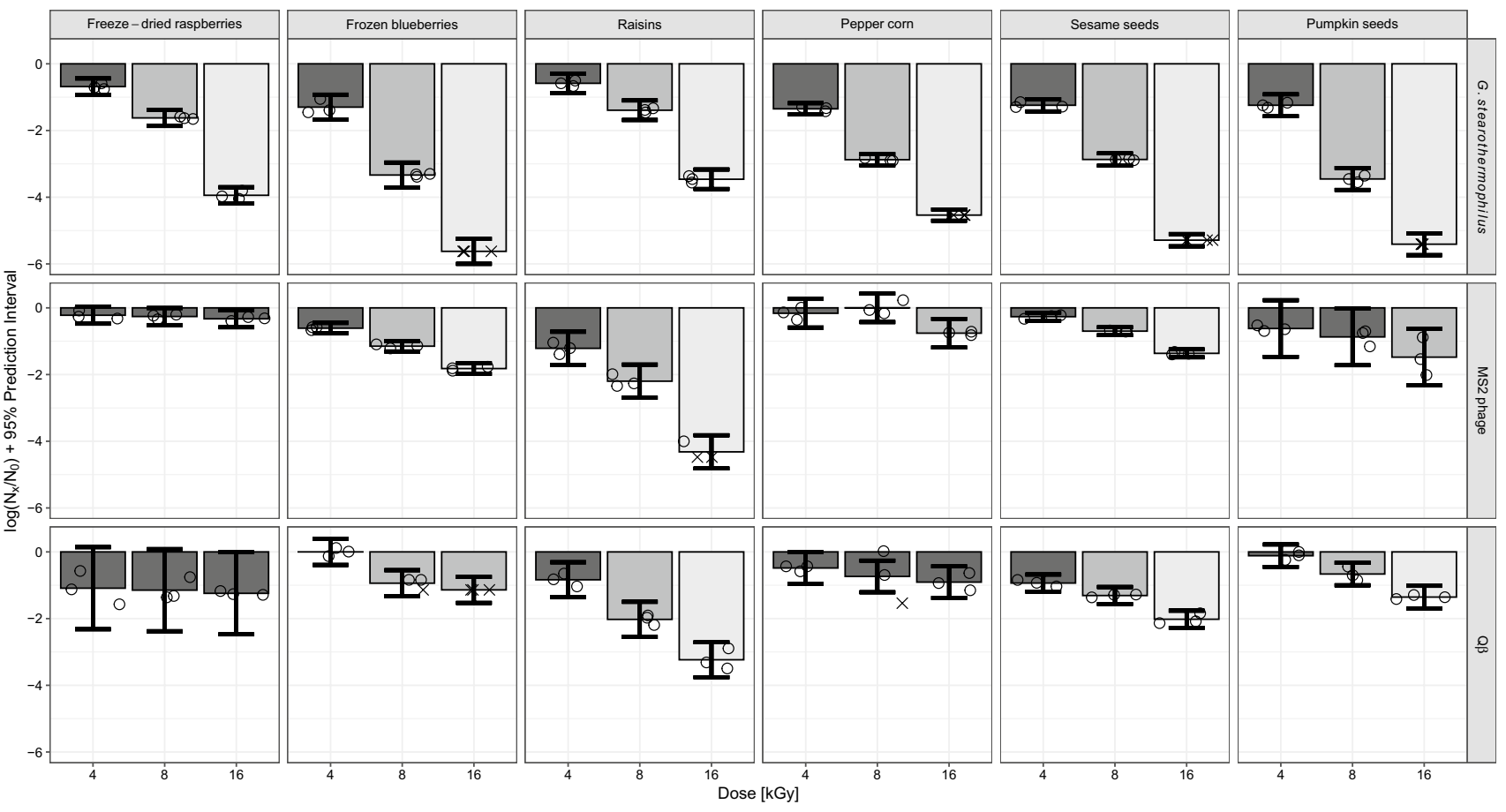

Fig. 2 Mean inactivation of G. stearothermophilus spores, MS2 and $\mathrm{Q} \beta$ bacteriophages on freeze-dried raspberries, frozen blueberries, raisins, pepper corns, sesame seeds and pumpkin seeds treated with HEEB at 4, 8 and $16 \mathrm{kGy}$. Different shadings show significant differences in the mean $\log _{10}$ reductions between dosages within each microorganism-matrix combination. Bars represent $95 \%$ prediction

\section{Inactivation of G. stearothermophilus Spores and MS2 During LEEB on Frozen Blueberries}

Geobacillus stearothermophilus spores and MS2 intervals. Open circles represent single values, crosses represent single values below the maximum measurable $\log _{10}$ reduction. Notice that since a common variance has been computed for a given microorganism-matrix combination, all the prediction intervals for that combination have the same width. Supplementary Table 2 contains the raw data used to generate Fig. 2
Fig. 3 Mean inactivation of G. stearothermophilus spores, HAV and MS2 bacteriophage on frozen blueberries treated with LEEB at $16 \mathrm{kGy}$ in comparison with corresponding reduction generated by HEEB at 8 and $16 \mathrm{kGy}$. Open circles represent single values, crosses represent single values below the maximum measurable $\log _{10}$ reduction

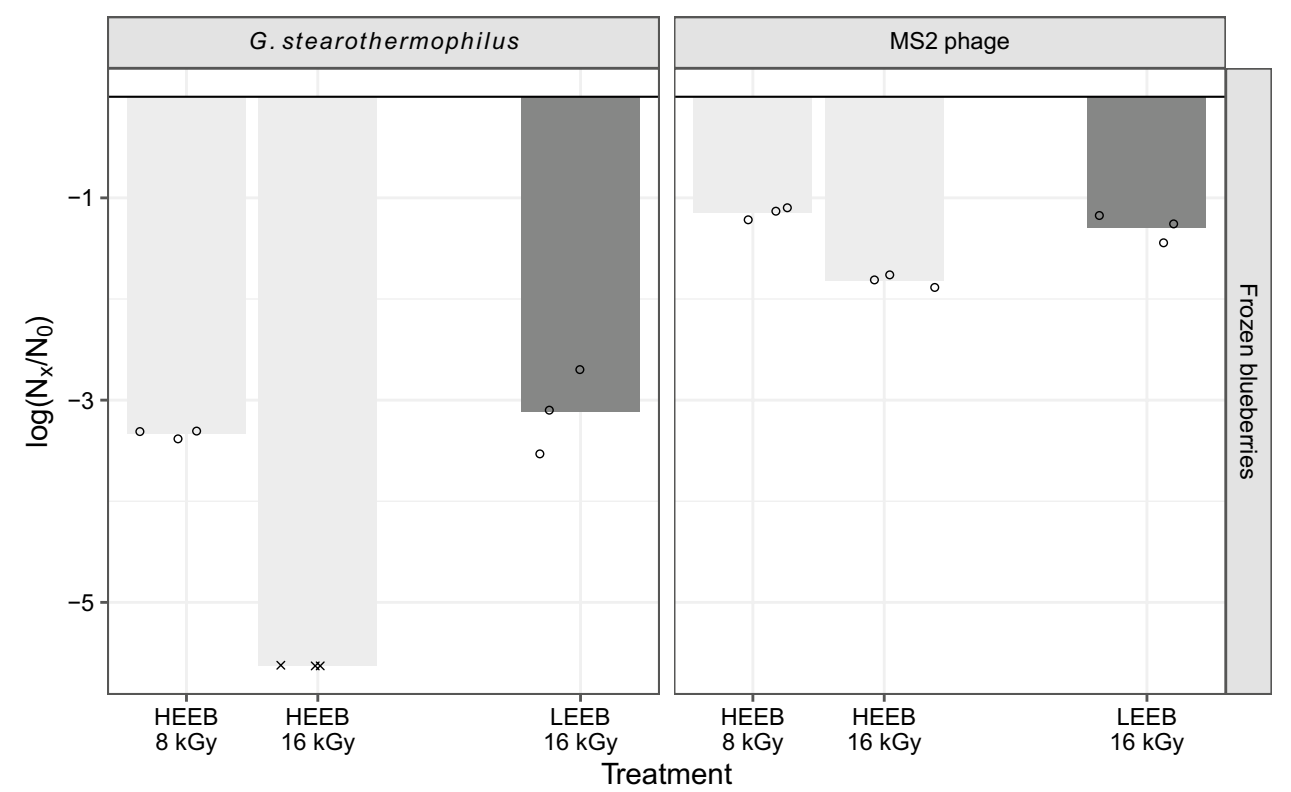

bacteriophage on frozen blueberries were treated in the industrial Bühler AG Laatu system, as a proof of concept for validation of HAV and $\mathrm{hNoV}$ inactivation by LEEB. In Fig. 3, the LEEB results obtained from this experiment are 
compared with the data generated by HEEB. As the LEEB experiment was a proof of concept with a low amount of data, we did not carry out statistical analysis on these data points. Mean reductions of $3.1 \log _{10}$ and $1.3 \log _{10}$ were measured after the LEEB surface treatment for G. stearothermophilus spores and MS2, respectively (Fig. 3). These values were similar to the $\log _{10}$ reductions determined for both organisms in the HEEB trials at $8 \mathrm{kGy}\left(3.3 \log _{10}\right.$ for G. stearothermophilus spores and $1.1 \log _{10}$ for MS2). The similarity of the $\log _{10}$ reductions of the surrogates observed on frozen blueberries after a LEEB treatment at $16 \mathrm{kGy}$ and a HEEB treatment at $8 \mathrm{kGy}$, suggests that if HAV underwent a LEEB treatment at $16 \mathrm{kGy}$, we might expect a reduction similar to the one determined for the HEEB treatment at $8 \mathrm{kGy}$ (Fig. 1). Thus, we expect to see a $\log _{10}$ reduction of 1.4 on frozen blueberries for HAV treated by LEEB at $16 \mathrm{kGy}$.

\section{Discussion}

The results of the present study represent a proof of concept for validation of HAV and hNoV inactivation by LEEB, using the industrial Bühler AG Laatu system, the only industrial scale system currently available to the food industry. As this system is an open system not allowing safe handling of pathogens, the HEEB technology was used with inoculated and packaged food matrices to compare reduction of $\mathrm{HAV}$ and $\mathrm{hNoV}$ with potential surrogates. The HEEB trials generated new data on the effect electron beam irradiation has on different viruses-food matrix combinations. Overall, this study demonstrates the high resistance of HAV to electron beam irradiation and an even higher resistance of MNV (Fig. 1). As different amino acids vary in their susceptibility to irradiation, crosslinking of proteins during irradiation may damage portions of the capsid involved in receptor binding to varying degrees and may explain differences in susceptibility observed between different viruses (Hume et al. 2016; Stewart 2001). A comparison with hNoV is needed in the future to determine which $\mathrm{hNoV}$ surrogate can reliably be used as surrogate to evaluate the effect of electron beam irradiation. Using the porcine gastric mucin magnetic bead (PGM-MB) binding assay, the level of hNoV and TV RNA decreased by $>1 \log _{10}$ and approximately 2 $\log _{10}$, respectively, following a $12.2 \mathrm{kGy}$ HEEB treatment on whole strawberries (DiCaprio et al. 2016). In comparison, at $8 \mathrm{kGy}$ for MNV our results reveal $\log _{10}$ reductions of 0.76 and $1.35 \log _{10}$ on frozen blueberries and raisins, respectively. As shown by Ettayebi and coauthors using the stem cell derived human enteroids assay, exposure to gamma irradiation of hNoV GII.3 and GII.4 stool suspensions inactivated the viruses, but at this point in time no data are available with this system on food matrices (Ettayebi et al. 2016).
As reported by others, spores of G. stearothermophilus are highly resistant against irradiation and showed a higher resistance during LEEB than the spores of B. pumilus which is often suggested to be the biological indicator for irradiation sterilization (Van Gerwen et al. 1999; Zhang et al. 2018). Therefore, G. stearothermophilus spores were included in this study to compare their resistance to electron beam with the one of HAV. We observed a higher susceptibility of G. stearothermophilus to HEEB compared to HAV (Figs. 1, 2). The data generated in this study for $G$. stearothermophilus is new and valuable for future industrial validation studies. It cannot be directly compared with the data present in the literature, as most studies have determined $D_{10}$ values for electron beam treatments of $B$. pumilus and $B$. subtilis and to a lesser extent $G$. stearothermophilus spores not inoculated onto any food matrix (Tallentire et al. 2010; Zhang et al. 2020, 2018). Nevertheless, the $D$-value of $3.1 \mathrm{kGy}$ at $200 \mathrm{keV}$ measured by Zhang and coauthors corresponds to a $2.6 \log _{10}$ reduction at $8 \mathrm{kGy}$ which is similar to the reduction of $2.87 \log _{10}$ we measured on peppercorn and sesame seeds. Additionally, the food matrix can have opposite effects on different microorganisms. For example, on raisins $G$. stearothermophilus showed a lower inactivation than on the other matrices. Viruses, on the other hand, generally showed higher inactivation on raisins compared to the other matrices, showing that extrapolation of effects from one matrix to another should not be attempted.

Low performance of the methods used to recover the viruses and the bacteriophages impacted only a small part of the results, as the maximum measurable $\log _{10}$ reductions were in most cases sufficient to draw meaningful conclusions. G. stearothermophilus spores showed good recoveries on every matrix, whereas enteric viruses and bacteriophages displayed poor recovery rates. For enteric viruses, such as HAV and MNV, this was expected as the methods available for the detection of viruses are well known for their complexities and low recovery efficiencies (Li et al. 2018; Mäde et al. 2013; Perrin et al. 2015). In ISO 15,216, the minimal recovery efficiency required is $1 \%$ but this requirement is often not reached ( $\mathrm{Li}$ et al. 2018). For example, Perrin and coauthors reported a recovery of $0.5 \%$ of $\mathrm{MNV}$ on raspberries and Hida and coauthors reported MNV recovery efficiencies of $0.06 \%$ and $0.41 \%$ on lettuce and grapes, respectively (Hida et al. 2018; Perrin et al. 2015). The low recovery efficiencies obtained with the bacteriophages were more surprising as the method used is in principle simple and easy to apply. Shim and coauthors showed a correlation between the stronger adhesion and the lower recoveries of MS2 on PVC, therefore one hypothesis of this low performance could be a strong adhesion of the bacteriophages on the matrices, impacting the recovery rates (Shim et al. 2017). One explanation of this stronger adhesion could be the time between the inoculation and the analysis, as shown by Shim 
and coauthors who extended this time from $1 \mathrm{~min}$ to $24 \mathrm{~h}$ to allow stronger MS2 attachment for their study (Shim et al. 2017).

The similarity of the $\log _{10}$ reductions observed between HEEB at $8 \mathrm{kGy}$ and LEEB at $16 \mathrm{kGy}$ for both G. stearothermophilus and MS2 on frozen blueberries may be due to several factors, one of them being the difference in the penetration depth of electrons between HEEB and LEEB. The HEEB measured dose is applied to the full depth of the food matrix. The LEEB dose is a surface and subsurface measurement, where the average absorbed dose along the food matrix is depending on the depth dose distribution at a given energy, the matrix density and its chemical composition (Shim et al. 2017). In the current study the beam penetration depth for frozen blueberries was not determined, but in a matrix with water density at $250 \mathrm{keV}$, the absorbed dose is maximum at the surface while it exponentially drops to zero at approximately $580 \mu \mathrm{m}$. If a certain number of microorganisms are beyond the penetration ability of the electrons (at $250 \mathrm{keV}$ ) they will not be inactivated. Additionally, it has been hypothesized that lower $D_{10}$ value for HEEB versus LEEB treatments may be due to the additional inactivation effect of reactive oxygen species if atmospheres during the treatments contain different amounts of $\mathrm{O}_{2}$. Thus, when comparing HEEB and LEEB systems, the focus should not be on comparing the dosimetry measurements. Instead, the impact of achieving the intended microbial reduction and the impact on food quality, such as nutrients and sensory attributes, should be evaluated. The quality of the HEEB and LEEB treated foods in this study were evaluated using sensory attributes. There were no visible differences between HEEB and LEEB treated foods in this study and detailed sensory profiles and chemical properties are described elsewhere (Aisala et al. in preparation).

Based on our results for G. stearothermophilus and MS2, a $\log _{10}$ reduction of 1.4 can be expected for HAV on frozen blueberries treated by LEEB at $16 \mathrm{kGy}$, indicating that LEEB processing cannot completely eliminate the risk of viral illness on berries and emphasizes the critical importance of adhering to stringent application of hygiene control systems that limit enteric virus contamination at primary production. In addition, the list of foods allowed for irradiation treatment in the EU currently comprises only dried aromatic herbs, spices and vegetable seasonings and not berries, but this may change in the future, as a legislative evaluation related to the irradiation of food and food ingredients is ongoing within the EU (Anonymous 2020).

Supplementary Information The online version contains supplementary material available at https://doi.org/10.1007/s12560-021-09463-3.

Acknowledgements We gratefully acknowledge Conrad Guenthard (LEONI Studer AG irradiation centre in Daeniken, Switzerland) for support with the HEEB trial setup and the dosimetry measurements, the Bühler AG team for the LEEB trials and dosimetry measurements and Mireille Moser for initial support with data analysis.

Author Contributions SZ was activity leader within EIT Food project ID19087 and supervised the study. SZ and SB designed the study, prepared and tested the samples and wrote the manuscript. LG performed the statistical analyses of the study and wrote the manuscript. TP prepared, tested the samples and helped to write the manuscript.

Funding This research was conducted under EIT Food project ID19087with funding from the European Institute of Innovation and Technology (EIT), a body of the European Union, under Horizon 2020, the EU Framework Program for Research and Innovation of the EIT Food project ID19087.

\section{Compliance with Ethical Standards}

Conflict of Interest The authors declare that they have no conflict of interest.

Open Access This article is licensed under a Creative Commons Attribution 4.0 International License, which permits use, sharing, adaptation, distribution and reproduction in any medium or format, as long as you give appropriate credit to the original author(s) and the source, provide a link to the Creative Commons licence, and indicate if changes were made. The images or other third party material in this article are included in the article's Creative Commons licence, unless indicated otherwise in a credit line to the material. If material is not included in the article's Creative Commons licence and your intended use is not permitted by statutory regulation or exceeds the permitted use, you will need to obtain permission directly from the copyright holder. To view a copy of this licence, visit http://creativecommons.org/licenses/by/4.0/.

\section{References}

Alegbeleye, O. O., Singleton, I., \& Sant'Ana, A. S. (2018). Sources and contamination routes of microbial pathogens to fresh produce during field cultivation: A review. Food Microbiology, 73, 177-208.

Anonymous. (2018). Swift investigation of hepatitis A outbreak facilitated withdrawal of dates. Copenhagen: Statens Serum Institute.

Anonymous. (2020). Evaluation of the EU legal framework on food irradiation. Brussels: EU.

Bidawid, S., Farber, J. M., \& Sattar, S. A. (2000). Inactivation of hepatitis A virus (HAV) in fruits and vegetables by gamma irradiation. International Journal of Food Microbiology, 57, 91-97.

Bosch, A., Gkogka, E., Le Guyader, F. S., Loisy-Hamon, F., Lee, A., van Lieshout, L., et al. (2018). Foodborne viruses: Detection, risk assessment, and control options in food processing. International Journal of Food Microbiology, 285, 110-128.

Butot, S., Zuber, S., \& Baert, L. (2014). Sample preparation prior to molecular amplification: Complexities and opportunities. Current Opinion in Virology, 4, 66-70.

Butot, S., Cantergiani, F., Moser, M., Jean, J., Lima, A., Michot, L., et al. (2018). UV-C inactivation of foodborne bacterial and viral pathogens and surrogates on fresh and frozen berries. International Journal of Food Microbiology, 275, 8-16.

De Lara, J., Fernández, P. S., Periago, P. M., \& Palop, A. (2002). Irradiation of spores of Bacillus cereus and Bacillus subtilis with electron beams. Innovative Food Science and Emerging Technologies, 3, 379-384. 
Delbeke, S., Ceuppens, S., Jacxsens, L., \& Uyttendaele, M. (2015). Survival of salmonella and escherichia coli O157:H7 on strawberries, basil, and other leafy greens during storage. Journal of Food Protection, 78, 652-660.

DiCaprio, E., Phantkankum, N., Culbertson, D., Ma, Y., Hughes, J. H., Kingsley, D., et al. (2016). Inactivation of human norovirus and Tulane virus in simple media and fresh whole strawberries by ionizing radiation. International Journal of Food Microbiology, 232, 43-51.

DiCaprio, E., Ye, M., Chen, H., \& Li, J. (2019). Inactivation of human norovirus and tulane virus by high pressure processing in simple mediums and strawberry puree. Frontiers in Sustainable Food Systems. https://doi.org/10.3389/fsufs.2019.00026.

Espinosa, A. C., Jesudhasan, P., Arredondo, R., Cepeda, M., MazariHiriart, M., Mena, K. D., \& Pillai, S. D. (2012). Quantifying the reduction in potential health risks by determining the sensitivity of poliovirus type 1 chat strain and rotavirus SA-11 to electron beam irradiation of iceberg lettuce and spinach. Applied and Environmental Microbiology, 78, 988-993.

Ethelberg, S., Lisby, M., Bottiger, B., Schultz, A. C., Villif, A., Jensen, T., et al. (2010). Outbreaks of gastroenteritis linked to lettuce, Denmark, January 2010. Euro Surveillance, 15, 19484.

Ettayebi, K., Crawford, S. E., Murakami, K., Broughman, J. R., Karandikar, U., Tenge, V. R., et al. (2016). Replication of human noroviruses in stem cell-derived human enteroids. Science, 353, 1387-1393.

Etter, D., Rupp, A., Prange, A., \& Drissner, D. (2018). Inactivation of mould spores in a model system and on raisins by low-energy electron beam. Food Control, 92, 357-361.

Fan, X., Sokorai, K., Weidauer, A., Gotzmann, G., Rögner, F. H., \& Koch, E. (2017). Comparison of gamma and electron beam irradiation in reducing populations of $E$. coli artificially inoculated on mung bean, clover and fenugreek seeds, and affecting germination and growth of seeds. Radiation Physics and Chemistry, 130, 306-315.

Farkas, J. (1998). Irradiation as a method for decontaminating food: A review. International Journal of Food Microbiology, 44, 189-204.

Farkas, J. (2006). Irradiation for better foods. Trends in Food Science and Technology, 17, 148-152.

Gryczka, U., Migdał, W., \& Bułka, S. (2018). The effectiveness of the microbiological radiation decontamination process of agricultural products with the use of low energy electron beam. Radiation Physics and Chemistry, 143, 59-62.

Hayashi, T., Takahashi, Y., \& Todoriki, S. (1998). Sterilization of foods with low-energy electrons ('Soft-electrons'). Radiation Physics and Chemistry, 52, 73-76.

Hertwig, C., Meneses, N., \& Mathys, A. (2018). Cold atmospheric pressure plasma and low energy electron beam as alternative nonthermal decontamination technologies for dry food surfaces: A review. Trends in Food Science and Technology, 77, 131-142.

Hida, K., Papafragkou, E., \& Kulka, M. (2018). Testing for human norovirus and recovery of process control in outbreak-associated produce items. Journal of Food Protection, 81, 105-114.

Hume, A. J., Ames, J., Rennick, L. J., Duprex, W. P., Marzi, A., Tonkiss, J., \& Mühlberger, E. (2016). Inactivation of RNA viruses by gamma irradiation: A study on mitigating factors. Viruses, 8, 204.

ISO. (1995). ISO 10705-1:1995: Water quality? Detection and enumeration of bacteriophages? Part 1: Enumeration of F-specific RNA bacteriophages. Geneva: ISO.

ISO. (2013). ISO/ASTM 51261:2013: Practice for calibration of routine dosimetry systems for radiation processing. Geneva: ISO.

ISO. (2017). ISO 15216-1:2017: Microbiology of the food chain: Horizontal method for determination of hepatitis A virus and norovirus in food using real-time RT-PCR: Part 1: Method for quantification. Geneva: ISO.
Kikuchi, O. K., Todoriki, S., Saito, M., \& Hayashi, T. (2003). Efficacy of soft-electron (low-energy electron beam) for soybean decontamination in comparison with gamma-rays. Journal of Food Science, 68, 649-652.

Kingsley, D. H., \& Chen, H. (2009). Influence of pH, salt, and temperature on pressure inactivation of hepatitis A virus. International Journal of Food Microbiology, 130, 61-64.

Knorr, D., \& Watzke, H. (2019). Food processing at a crossroad. Frontiers in Nutrition, 6, 85.

Li, D., Butot, S., Zuber, S., \& Uyttendaele, M. (2018). Monitoring of foodborne viruses in berries and considerations on the use of RT-PCR methods in surveillance. Food Control, 89, 235-240.

Mäde, D., Trübner, K., Neubert, E., Höhne, M., \& Johne, R. (2013). Detection and typing of norovirus from frozen strawberries involved in a large-scale gastroenteritis outbreak in Germany. Food and Environmental Virology, 5, 162-168.

Miranda, R. C., \& Schaffner, D. W. (2019). Virus risk in the food supply chain. Current Opinion in Food Science, 30, 43-48.

Moosekian, S. R., Jeong, S., Marks, B. P., \& Ryser, E. T. (2012). X-ray irradiation as a microbial intervention strategy for food. Annual Review of Food Science and Technology, 3, 493-510.

Perrin, A., Loutreul, J., Boudaud, N., Bertrand, I., \& Gantzer, C. (2015). Rapid, simple and efficient method for detection of viral genomes on raspberries. Journal of Virological Methods, 224, 95-101.

Pillai, S. D., \& Shayanfar, S. (2017). Electron beam technology and other irradiation technology applications in the food industry. Topics in Current Chemistry, 375, 6.

Praveen, C., Dancho, B. A., Kingsley, D. H., Calci, K. R., Meade, G. K., Mena, K. D., \& Pillai, S. D. (2013). Susceptibility of murine norovirus and hepatitis a virus to electron beam irradiation in oysters and quantifying the reduction in potential infection risks. Applied and Environmental Microbiology, 79, 3796-3801.

R-Core Team. (2019). R: A language and environment for statistical computing. Vienna: R-Core Team.

Ravindran, R., \& Jaiswal, A. K. (2019). Wholesomeness and safety aspects of irradiated foods. Food Chemistry, 285, 363-368.

Sanglay, G. C., Li, J., Uribe, R. M., \& Lee, K. (2011). Electron-beam inactivation of a norovirus surrogate in fresh produce and model systems. Journal of Food Protection, 74, 1155-1160.

Shim, J., Stewart, D. S., Nikolov, A. D., Wasan, D. T., Wang, R., Yan, R., \& Shieh, Y. C. (2017). Differential MS2 interaction with food contact surfaces determined by atomic force microscopy and virus recovery. Applied and Environmental Microbiology, 83, e01881-e01917.

Stewart, E. M. (2001). Food irradiation chemistry. In R. Molins (Ed.), Food irradiation: Principles and applications (pp. 37-76). New York: Wiley.

Tallentire, A., Miller, A., \& Helt-Hansen, J. (2010). A comparison of the microbicidal effectiveness of gamma rays and high and low energy electron radiations. Radiation Physics and Chemistry, 79, 701-704.

Tavoschi, L., Severi, E., Niskanen, T., Boelaert, F., Rizzi, V., Liebana, E., et al. (2015). Food-borne diseases associated with frozen berries consumption: A historical perspective, European Union, 1983 to 2013. Eurosurveillance Weekly, 20, 21193.

Tetra Pak (2019). Tetra Pak E3 with eBeam. Retrieved from https:// www.tetrapak.com/tetra-pak-e3-ebeam. Accessed 29 Oct 2020.

Thebault, A., Teunis, P. F., Le Pendu, J., Le Guyader, F. S., \& Denis, J. B. (2013). Infectivity of GI and GII noroviruses established from oyster related outbreaks. Epidemics, 5, 98-110.

Tree, J. A., Adams, M. R., \& Lees, D. N. (2005). Disinfection of feline calicivirus (a surrogate for Norovirus) in wastewaters. Journal of Applied Microbiology, 98, 155-162.

Van Gerwen, S. J. C., Rombouts, F. M., Riet, K. V., \& Zwietering, M. H. (1999). A data analysis of the irradiation parameter for bacteria 
and spores under various conditions. Journal of Food Protection, 62, 1024-1032.

Wiedenmann, A., Fischer, B., Straub, U., Wang, C.-H., Flehmig, B., \& Schoenen, D. (1993). Disinfection of hepatitis a virus and MS-2 coliphage in water by ultraviolet irradiation: Comparison of UVsusceptibility. Water Science and Technology, 27, 335-338.

Zhang, Y., Huber, N., Moeller, R., Stülke, J., Dubovcova, B., Akepsimaidis, G., et al. (2020). Role of DNA repair in Bacillus subtilis spore resistance to high energy and low energy electron beam treatments. Food Microbiology, 87, 103353.
Zhang, Y., Moeller, R., Tran, S., Dubovcova, B., Akepsimaidis, G., Meneses, N., et al. (2018). Geobacillus and bacillus spore inactivation by low energy electron beam technology: Resistance and influencing factors. Frontiers in Microbiology, 9, 2790.

Publisher's Note Springer Nature remains neutral with regard to jurisdictional claims in published maps and institutional affiliations. 